\title{
Pulmonary Melioidosis Masquerading as Tuberculosis: A Case Report
}

\author{
Shital Patil ${ }^{1 *}$, Gajanan Gondhali ${ }^{2}$
}

${ }^{1}$ Associate Professor, Pulmonary Medicine, MIMSR Medical College, Latur, Maharashtra, INDIA

${ }^{2}$ Associate Professor, Internal Medicine, MIMSR Medical College, Latur, Maharashtra, INDIA

*Corresponding Author: drsvpatil1980@gmail.com

Citation: Patil S, Gondhali G. Pulmonary Melioidosis Masquerading as Tuberculosis: A Case Report. Electron J Gen Med. 2021;18(5):em310. https://doi.org/10.29333/ejgm/11064

\section{ARTICLE INFO}

Received: 14 Mar. 2021

Accepted: 19 Jun. 2021

\begin{abstract}
Tuberculosis is most common chronic lung infection in India and it will present radiologically from nodules with branching lines called as 'tree in bud' to consolidations. Radiological presentations in various chronic lung diseases are overlapping and microbiological confirmation is must rather than giving empirical ant-tuberculosis treatment. Melioidosis is bacteriological infection causing similar clinical and radiological syndrome mimicking tuberculosis. In this case report, 70-year female presented with chronic respiratory illness with constitutional symptoms like cough, low grade fever, weight loss and anorexia and HRCT thorax imaging showing progressive pathologies as tree in bud to consolidations over 3 months duration. She had received anti-tuberculosis treatment empirically for 3 months and was having clinical and radiological deterioration. Bronchoscopy guided bronchoalveolar lavage (BAL) analysis for Gene Xpert MTB/RIF and Bacterial culture suggestive of Melioidosis as cause for her chronic respiratory illness. She was treated as per antibiotic protocol for Melioidosis and shown complete clinical and radiological response.
\end{abstract}

Keywords: tuberculosis, Melioidosis, tree in bud, BAL, gene Xpert MTB/RIF

\section{INTRODUCTION}

Melioidosis is caused by B. pseudomallei, a Gram-negative bacilli commonly found in soil or contaminated water, and it is an endemic disease in northern Australia and Southeast Asian countries, in particular Thailand and Malaysia [1]. The clinical spectrum of melioidosis is complex and wide-ranging, and includes latent infection, subacute pneumonia, local cutaneous lesions, focal organ abscess, musculoskeletal infection, bacteremia and lethal fulminant pneumonia $[2,3]$. High index of suspicion is must while managing these chronic infections being high mortality and overlap with other tropical respiratory infections like tuberculosis. The aim of this case is to create more awareness among health care professionals regarding melioidosis may be the etiological factor for "chronic respiratory infections with Lung infiltrates" in tropical setting.

\section{CASE SUMMARY}

70-year female, farmer by occupation, presented to emergency room with history of constitutional symptoms like dry cough, low grade fever and weight loss in the past 3 months, anorexia in the past 2 months, she was on antituberculosis treatment in the past 3 months and referred to our center for clinical and radiological worsening.

\section{Clinical Examination Documented}

Pallor, pitting pedal edema, Heart rate-100/min BP-100/60 mmhg,
Respiratory rate-30/min, $\mathrm{PsO} 2-88 \%$ room air, $93 \%$ on nasal canula oxygen@2 liters/min,

Respiratory system examination revealed crepitations in right infraclavicular and mammary area.

\section{Laboratory Examination Documented}

Hemoglobin-10 gm\% total white blood cells- 19000/mm Polymorphs-95\%,

CRP-190 units/liter liver and kidney functions- normal.

Presented to our unit with HRCT thorax images as: done 3 months back and images were reported as, right upper lobe nodular opacities with branching lines called as 'tree in bud opacities' (Figures 1 and 2) and small airspace opacities in right upper lobe with tree in bud opacities (Figure 3).

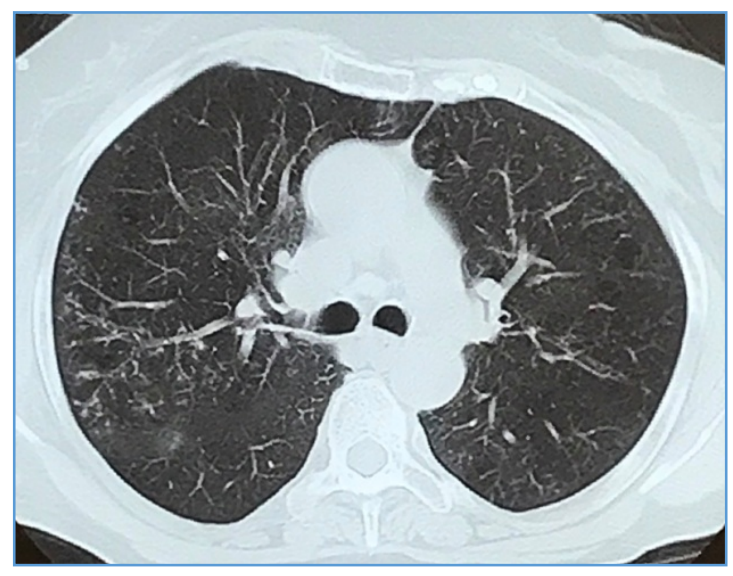

Figure 1. CT thorax- tree in bud pattern 


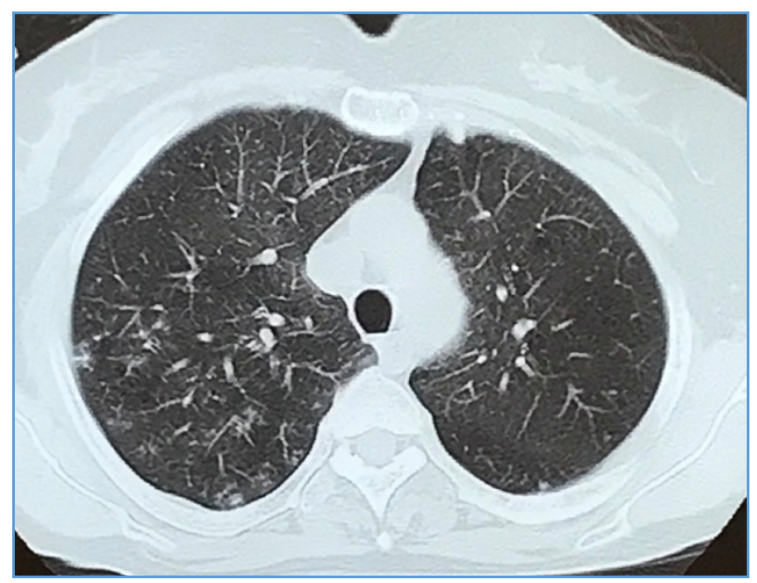

Figure 2. CT thorax- branching lines with air space opacities

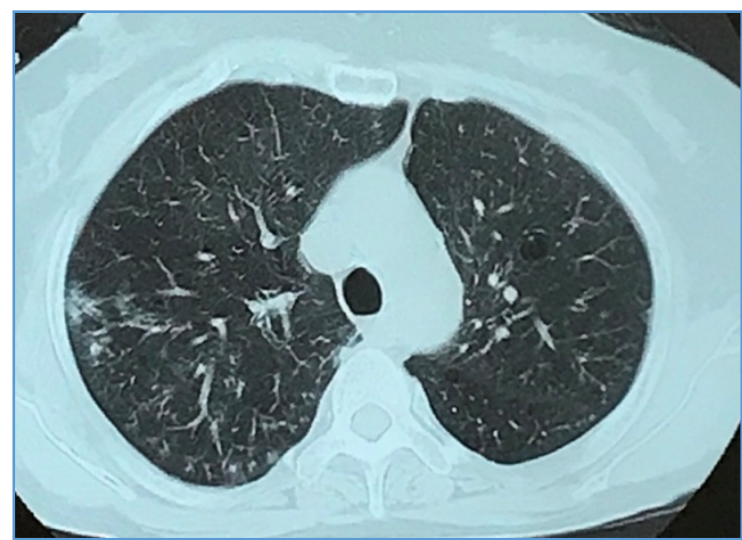

Figure 3. CT thorax- air space opacities

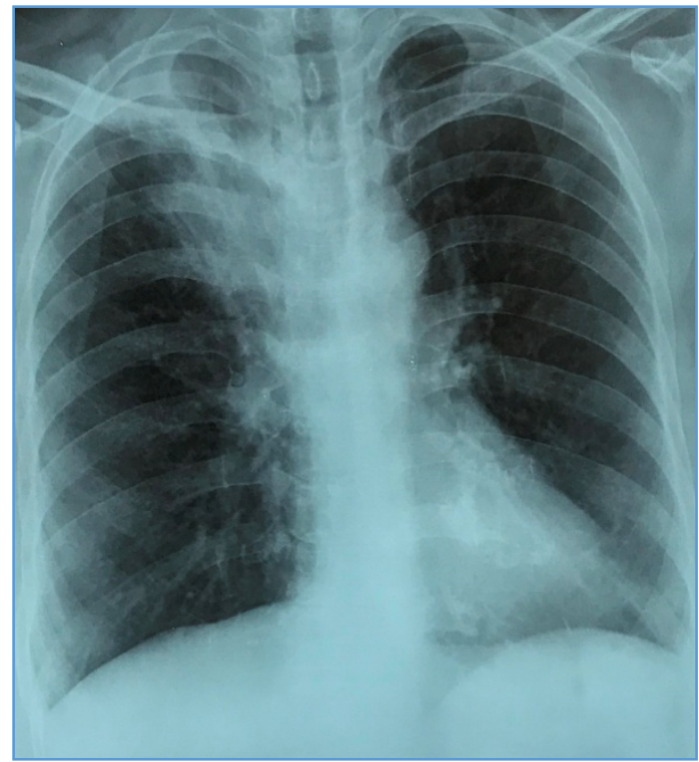

Figure 4. Chest x-ray PA- Paratracheal opacity

She had received anti-tuberculosis treatment for 3 months and shown clinical and radiological deterioration. She was referred to our center for clinical excellence, brought chest $x$ ray showing airspace opacity occupying right Paratracheal area and showing right upper zone opacification or consolidation (Figure 4). We have continued antibiotics with anti-tuberculosis treatment for 10 days, repeated chest $\mathrm{x}$-ray

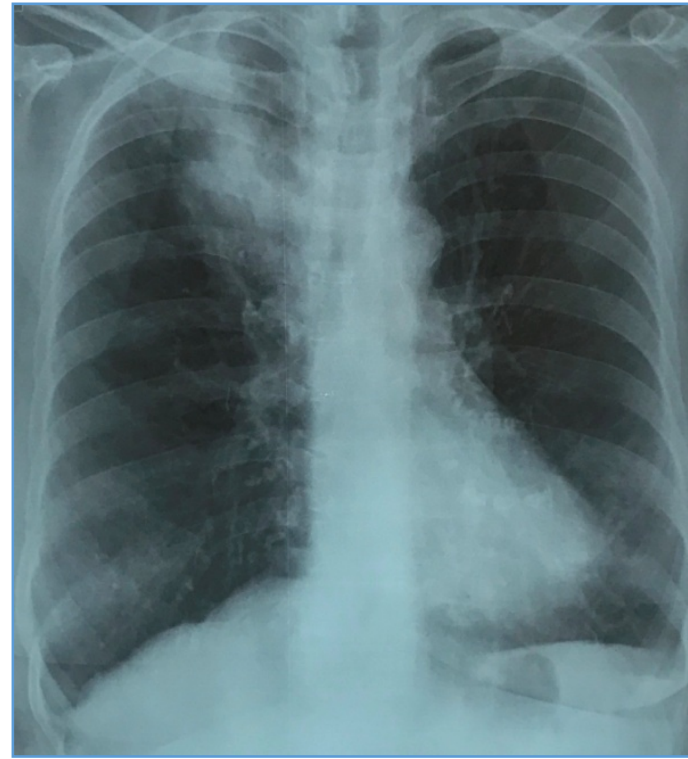

Figure 5. Chest x-ray PA- paratracheal air space opacity

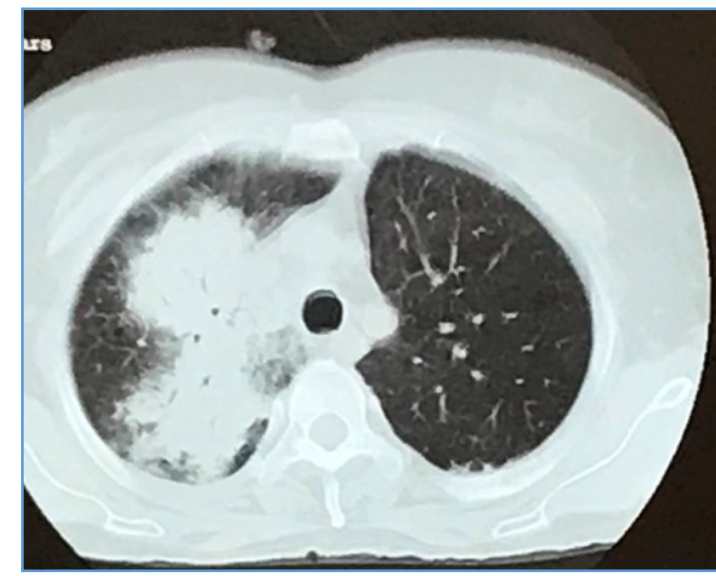

Figure 6. CT thorax-consolidation with air bronchogram

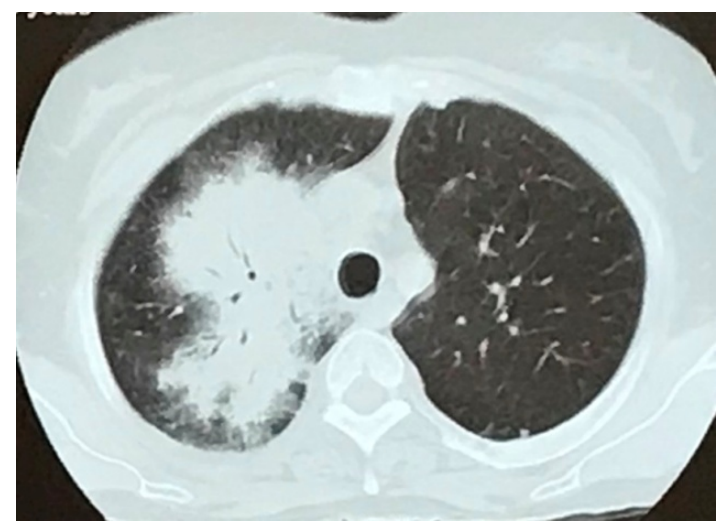

Figure 7. CT thorax- consolidation with necrosis

shown partial response to treatment and resolution in opacity on chest radiograph (Figure 5 ).

As response to treatment is delayed, we have performed HRCT thorax, showing airspace opacities with lucencies (Figure 6) and air bronchogram (Figure 7) in right upper lobe anterior segment (Figure 8) suggestive of consolidation right upper lobe secondary to chronic respiratory infection or inflammation. 


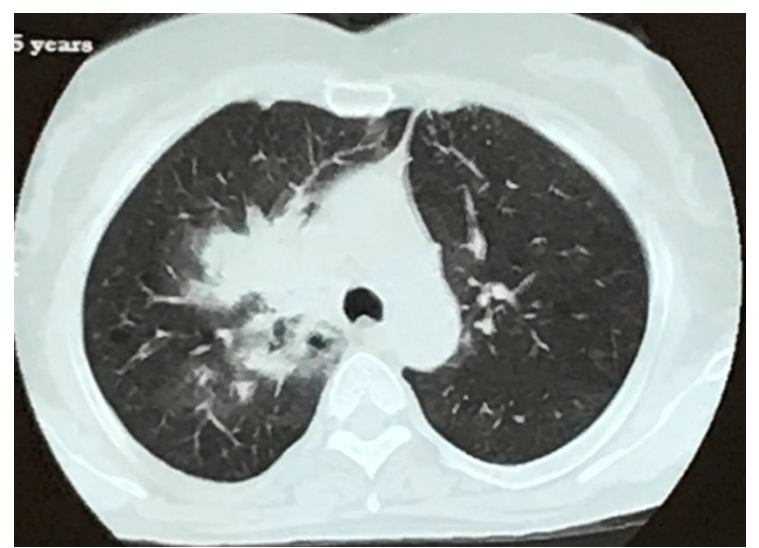

Figure 8. CT thorax-consolidation

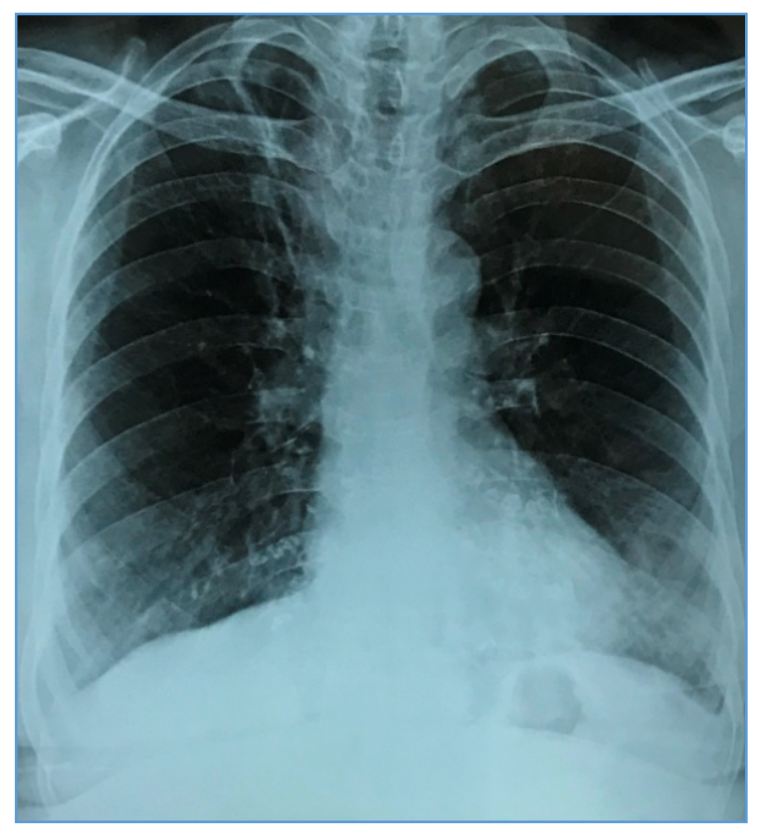

Figure 9. Chest x-ray PA- resolving consolidation

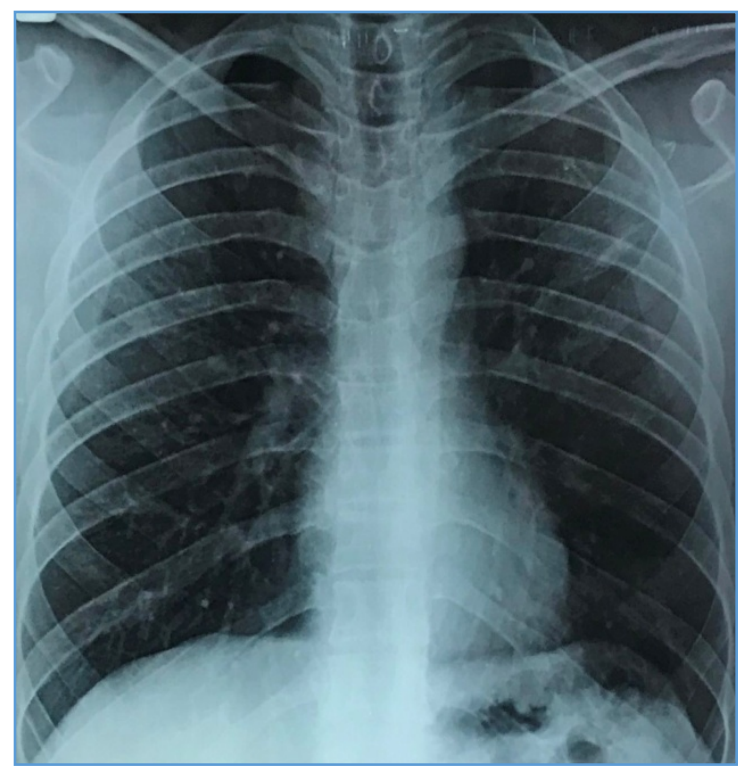

Figure 10. Chest X-ray PA- normal lung parenchyma

As patient is unable to produce sputum in spite of sputum induction, we have performed Fiberoptic videobronchoscopy with oxygen supplementation showing purulent secretions coming out from right upper lobe anterior segment, $80 \mathrm{ml}$ saline introduced bronchoscopically in right upper lobe anterior segment and broncho-alveolar lavage collected in three different aliquots, sent for cytology, Gene Xpert MTB/RIF and Bacterial culture respectively.

BAL cytology suggestive of Neutrophilic suppurative inflammation, negative for acid fast bacilli, gram stain suggestive of gram negative rods, Negative for fungal stains and negative for malignant cells.

BAL gene Xpert MTB/RIF negative for MTB genome.

BAL fluid culture grew 'Burkholderia pseudomallei' and antibiotic sensitivity pattern shown sensitive to amoxicillinclavulanate (co-amoxyclav), meropenem, ceftazidime.

We have started, Meropenum $1 \mathrm{gm}$ IV three times for 10 days, omitted anti-tuberculosis treatment, documented clinical recovery as fever subsides on day 3 of meropenem, appetite improved, shortness of breath decreased, vital parameters recovered with improvement I oxygen saturation as maintain spo $2>94 \%$ at room air.

Chest $x$-ray done on day 10 of treatment shown significant radiological response and clearance in opacification of right upper zone (Figure 9) and patient discharged to home with oral co-amoxyclav $1 \mathrm{gm}$ BID given for two weeks, oral doxycyclin $100 \mathrm{mg}$ BID for one month and trimethoprimsulphamethoxazole BID for six weeks to have total 3 months duration, and follow-up chest $x$-ray taken shown near complete resolution of right upper zone opacification (Figure 10).

We have followed up clinical and radiological details for 3 months of post discharge and documented no recurrence or relapse of pulmonary melioidosis and having normal post discharge parameters.

\section{DISCUSSION}

Melioidosis is caused by B. pseudomallei, a facultative intracellular Gram-negative saprophytic bacterium commonly found in soil or contaminated water [4]. It is a multiorgan infectious disease which can present with a wide spectrum of clinical presentations. The Darwin study found pneumonia to be the most common presentation of melioidosis in approximately half of cases. The proportion with pneumonia is much higher in those with chronic disease and those presenting after reactivation from a latent focus. Less common presentations include genitourinary infection (14\%), skin infection (13\%), bacteremia without evident focus (11\%), septic arthritis or osteomyelitis (4\%) and neurological melioidosis (3\%) [3].

Pneumonia is the commonest clinical presentation of melioidosis in all studies with around half of all melioidosis patients presenting with pneumonia. The proportion with pneumonia is much higher in those with chronic disease and those presenting after reactivation from a latent focus. In the Darwin prospective study, pneumonia was the primary diagnosis in 127 out of 252 cases (50\%) and an additional 21 cases $(8 \%)$ developed pneumonia after another primary diagnosis [5].

Patients with chronic pulmonary melioidosis have fevers, weight loss and a productive cough, sometimes with haemoptysis [6,7]. Pleuritic chest pain occurs in half [7]. Disease is often slowly progressive over months. It can also be 
remitting and relapsing over many years, but acute deterioration with septicaemia may also occur. Classically upper lobe changes with infiltrates and/or cavitation are seen on chest radiography, being present in 37 out of 39 (95\%) chronic cases in one study [7]. Initial chest radiography showed cavitary disease with or without infiltrates in 27 out of 39 (69\%) and infiltrates in 12 out of 39 (31\%) [103]. Pleural effusions were present in two out of 39, hilar adenopathy in only one out of 39 and only three out of 39 had bilateral disease. The cavities are usually single and thin walled and rarely contain an air-fluid level [6-8]. Computed tomography (CT) scan may show small cavities not evident on chest radiography [8]. There are numerous reports of chronic melioidosis being initially misdiagnosed as tuberculosis. Few studies $[9,10]$ have shown similar clinical and radiological presentations as documented in our case.

\section{Key Points}

1. Although Constitutional symptoms like cough, fever \& weight loss is common clinical scenario in tuberculosis, pulmonary Melioidosis may share similar clinical picture.

2. "Nodular opacities with tree in bud" is nonspecific finding to predict tuberculosis, although seen in majority. Many infective and inflammatory conditions are having similar radiological findings.

3. Progression of pulmonary infiltrates to consolidation, with constitutional symptoms; and in cases with unable to produce sputum, Bronchoscopy has significant role in diagnosis and management.

4. Bronchoscopy guided BAL has game changer role in these cases and BAL for Gene xpert MTB/RIF and bacterial culture is key investigations for final diagnosis.

5. In current trends, 'empirical ATT' should not started as 'radiological TB' as many of these cases may have other tropical infections like Melioidosis.

6. Pulmonary Melioidosis is chronic respiratory infection with similar, clinical and radiological presentations as like tuberculosis, and this differentiation is must as tuberculosis requires longer treatment up to six months while Melioidosis require shorter duration.

7. Lastly, for successful treatment outcome in "chronic respiratory infections with Lung infiltrates" clinician should follow standard acre of diagnosis and management with all possible methods including interventional pulmonary procedures like
Bronchoscopy and guided techniques like BAL Gene Xpert MTB/RIF and BAL culture.

Author contributions: All authors have sufficiently contributed to the study, and agreed with the results and conclusions.

Funding: No funding source is reported for this study.

Declaration of interest: No conflict of interest is declared by authors.

\section{REFERENCES}

1. Limmathurotsakul D, Golding N, Dance DA, Messina JP, Pigott DM, Moyes CL, et al. Predicted global distribution of Burkholderia pseudomallei and burden of melioidosis. Nat Microbiol 2016;1(1). https://doi.org/10.1038/nmicrobiol. 2015.8 PMCid:PMC4746747

2. White NJ. Melioidosis. Lancet 2003;361(9370):1715e22. https://doi.org/10.1016/S0140-6736(03)13374-0

3. Currie BJ, Ward L, Cheng AC. The epidemiology and clinical spectrum of melioidosis: 540 cases from the 20 Year Darwin prospective study. PLoS Neglected Trop Dis 2010;4(11):e900. https://doi.org/10.1371/journal.pntd. 0000900 PMid:21152057 PMCid:PMC2994918

4. Wiersinga WJ, Virk HS, Torres AG, Currie BJ, Peacock SJ, Dance DAB, et al. Melioidosis. Nat Rev Dis Prim 2018;4:17107. https://doi.org/10.1038/nrdp.2017.107 PMid:29388572 PMCid:PMC6456913

5. B.J. Currie. Melioidosis: an important cause of pneumonia in residents of and travellers returned from endemic regions. Eur Respir J 2003;22:542-50. https://doi.org/ 10.1183/09031936.03.00006203 PMid:14516149

6. Ip M, Osterberg LG, Chau PY, Raffin TA. Pulmonary melioidosis. Chest 1995;108:1420-4. https://doi.org/ 10.1378/chest.108.5.1420

7. Everett ED, Nelson RA. Pulmonary melioidosis. Observations in thirty-nine cases. Am Rev Respir Dis 1975;112:331-40.

8. Singcharoen T. CT findings in melioidosis. Australas Radiol 1989;33:376-8. https://doi.org/10.1111/j.1440-1673.1989. tb03316.x PMid:2633740

9. Tan RZ, Mohd Nor F, Shafie S, Tan LJ. Melioidosis mimicking miliary tuberculosis. Forensic Sci Med Pathol. 2019 Mar;15(1):151-4. https://doi.org/10.1007/s12024-018-00263 PMID:30293222

10. Vidyalakshmi K, Chakrapani M, Shrikala B, Damodar S, Lipika S, Vishal S. Tuberculosis mimicked by melioidosis. Int J Tuberc Lung Dis. 2008 Oct;12(10):1209-15. PMID:18812053. 